OPEN ACCESS

Edited by:

Preston E. Garraghty,

Indiana University Bloomington,

United States

Reviewed by:

Todd Mowery,

Rutgers, The State University

of New Jersey, United States

Merri J. Rosen,

Northeast Ohio Medical University,

United States

*Correspondence:

Yanqiu Feng

foree@smu.edu.cn

Condon Lau

condon.lau@cityu.edu.hk

†These authors share first authorship

Received: 18 January 2021 Accepted: 02 July 2021

Published: 12 August 2021

Citation:

Manno FAM, An Z, Kumar R,

Wu EX, He J, Feng Y and Lau C (2021) Structural Alterations in a Rat

Model of Short-Term Conductive Hearing Loss Are Associated With Reduced Resting State Functional

Connectivity

Front. Syst. Neurosci. 15:655172. doi: 10.3389/fnsys.2021.655172

\section{Structural Alterations in a Rat Model of Short-Term Conductive Hearing Loss Are Associated With Reduced Resting State Functional Connectivity}

\author{
Francis A. M. Manno' ${ }^{1 \dagger}$, Ziqi An ${ }^{2,3 \dagger}$, Rachit Kumar ${ }^{4,5}$, Ed X. Wu ${ }^{6,7}$, Jufang He ${ }^{8,9}$, \\ Yanqiu Feng ${ }^{2,3 *}$ and Condon Lau ${ }^{1 *}$
}

${ }^{1}$ Department of Physics, City University of Hong Kong, Hong Kong, SAR China, ${ }^{2}$ Guangdong Provincial Key Laboratory of Medical Image Processing, School of Biomedical Engineering, Southern Medical University, Guangzhou, China, ${ }^{3}$ Key Laboratory of Mental Health of the Ministry of Education, Guangdong-Hong Kong-Macao Greater Bay Area Center for Brain Science and Brain-Inspired Intelligence, Southern Medical University, Guangzhou, China, ${ }^{4}$ Perelman School of Medicine, University of Pennsylvania, Philadelphia, PA, United States, ${ }^{5}$ Medical Scientist Training Program, University of Pennsylvania, Philadelphia, PA, United States, ${ }^{6}$ Department of Electrical and Electronic Engineering, The University of Hong Kong, Hong Kong, SAR China, ${ }^{7}$ Laboratory of Biomedical Imaging and Signal Processing, The University of Hong Kong, Hong Kong, SAR China, ${ }^{8}$ Department of Neuroscience, City University of Hong Kong, Hong Kong, SAR China, ${ }^{9}$ Department of Biomedical Sciences, City University of Hong Kong, Hong Kong, SAR China

Conductive hearing loss $(\mathrm{CHL})$ results in attenuation of air conducted sound reaching the inner ear. How a change in air conducted sound alters the auditory system resulting in cortical alterations is not well understood. Here, we have assessed structural and functional magnetic resonance imaging (MRI) in an adult (P60) rat model of short-term conductive hearing loss (1 week). Diffusion tensor imaging (DTI) revealed fractional anisotropy (FA) and axial diffusivity alterations after hearing loss that circumscribed the auditory cortex (AC). Tractography found the lateral lemniscus tract leading to the bilateral inferior colliculus (IC) was reduced. For baseline comparison, DTI and tractography alterations were not found for the somatosensory cortex. To determine functional connectivity changes due to hearing loss, seed-based analysis (SBA) and independent component analysis (ICA) were performed. Short term conductive hearing loss altered functional connectivity in the $\mathrm{AC}$ and $\mathrm{IC}$, but not the somatosensory cortex. The results present an exploratory neuroimaging assessment of structural alterations coupled to a change in functional connectivity after conductive hearing loss. The results and implications for humans consist of structural-functional brain alterations following short term hearing loss in adults.

Keywords: conductive hearing loss, resting state functional connectivity, structural MRI, diffusion tensor imaging, rat - brain 


\section{INTRODUCTION}

\section{Impact of Conductive Hearing Loss}

Conductive hearing loss (CHL) results in the attenuation of air conducted sound (Willcox and Artz, 2007). Bilateral CHL produces air-conducted sound attenuation but does not raise bone-conducted thresholds (indicating no cochlear damage: Tucci et al., 1999). The loss of peripheral sound input (i.e., afferent) results in a dramatic decrease to inhibitory synaptic strength (GABA is decreased; Kotak et al., 2008, 2013) and increase in excitatory strength (Glutamate is increased - Kotak and Sanes, 1997; Kotak et al., 2005) in the lateral superior olive auditory pathway. Interestingly, the emergence of the inhibitory synaptic strength results in a critical period where deficits can be induced in juveniles, but not adults (Takesian et al., 2012). Early CHL induced by malleus removal results in task-specific behavioral threshold increases of $\approx 35$ decibel (dB) for sinusoidally amplitude modulated (AM) stimuli and increases of $\approx 40 \mathrm{~dB}$ in neural auditory brainstem responses to $100 \mu$ s clicks or $4 \mathrm{~ms}$ pure tones (Rosen et al., 2012). Additionally, developmental CHL animals display slower rates of acquisition for AM discrimination tasks due to an impaired ability to generalize newly introduced stimuli (von Trapp et al., 2017). The perceptual deficits found in CHL are likely due to aberrant processing in the auditory cortex (AC; i.e., decrease of inhibitory synaptic strength; Yao and Sanes, 2018, argue not in the auditory brainstem). Nevertheless, efferent pathways are also altered by CHL (Liberman et al., 2015). Here it was shown that the density of olivocochlear terminals in the cochlear epithelium is reduced (Liberman et al., 2015). We recognize the variety of studies in CHL complicate any general conclusions, as the majority of studies were conducted during development. The auditory system is greatly impacted due to CHL, yet investigations into structural-functional correlations in hearing loss are lacking, with studies concentrating either on structural or functional approaches.

\section{Animal Models of Hearing Loss Using Magnetic Resonance Imaging}

We have recently assessed the structural foundations of hearing loss across the lifespan in humans using a meta-analysis and meta-regression (Manno et al., 2021). In humans, we found that hearing loss not only affects auditory structures, but is brainwide, multi-focal, and impacts regions and tracts differently depending on auditory input and compensatory mechanisms. In rats, we have demonstrated acute and chronic noise exposure result in structural and functional changes in auditory structures from the midbrain to the cortex (Abdoli et al., 2016; Yang et al., 2018; Wong et al., 2020). In long-term passive noise exposure, diffusion tensor imaging (DTI), voxel-based statistics (VBS) revealed greater fractional anisotropy (FA) in the pyramidal tract and decreased FA in the tectospinal tract and trigeminothalamic tract (Abdoli et al., 2016). Acute noise exposure showed a reduction in midbrain sound-evoked responses as early as 1 week, as assessed using functional magnetic resonance imaging (fMRI) (Yang et al., 2018). Using a salicylate-induced model of hyperacusis, we found AC tone-evoked fMRI signals to 80 decibel sound pressure level (dB SPL) with 8,16 , and $32 \mathrm{kHz}$ stimuli during salicylate treatment were consistently larger than saline (indicating evidence of enhanced central gain; Wong et al., 2020). In contrast, the lateral lemniscus and inferior colliculus (IC) fMRI response obtained during salicylate treatment were similar to saline control values (Wong et al., 2020). Rodent models of hearing loss provide valuable information for establishing the basis of pathophysiology (Salvi et al., 2020) and further investigations should study the variety of hearing losses which occur in humans.

\section{Present Study Questions}

In the present experiments we sought to explore the structuralfunctional correlations of a rat model of CHL using neuroimaging techniques. Here, we used DTI and fMRI to determine how CHL alters the rat brain over a short duration. The present study was a repeated analysis self-controlled design, scanning rats prior to and 1-week after CHL induction. We ask the following questions: (1) what structural alterations can be revealed by DTI after CHL induction and (2) do these structural alterations result in functional deficits? The present study found reduced DTI metrics in the AC, reduced tract length and density of the lateral lemniscus as assessed by DTI, and reduced resting-state fMRI (rsfMRI) signal functional connectivity in the AC. Future studies should examine structural-functional relationships long term and therapeutic regimens to rehabilitate hearing loss models.

\section{MATERIALS AND METHODS}

\section{Animals Preparation}

Animals were prepared for fMRI experiments as described in our earlier studies (Lau et al., 2011, 2013, 2018; Cheung et al., 2012a,b; Lau et al., 2015a,b; Wong et al., 2017; Dong et al., 2018; Manno et al., 2019). All aspects of this study were approved by the Committee on the Use of Live Animals in Teaching and Research at the City University of Hong Kong. The present experiments consisted of behavioral assays and structural-functional MRI. For structural-functional MRI experiments, male Sprague-Dawley rats $(n=12 ; 250 \mathrm{~g}$, postnatal day 60$)$ were scanned before and 1 week after CHL induction (repeated analysis self-control design). Rodents were cage-housed under a constant $25^{\circ} \mathrm{C}$ temperature and $60-70 \%$ humidity. After surgery, the animals were returned to their home cages under warm conditions for recovery, and they were housed under a 12:12-h light/dark cycle in a temperaturecontrolled room with ad libitum access to food and water. Rodents were acclimated to the housing environment for at least one day prior to the experiment. Anti-inflammatory drugs were supplied in the water for 1 week. The experiments were conducted in a repeated analysis design where CHL was induced after an initial control period, and animals were subsequently used as their self-control for all experiments.

\section{Conductive Hearing Loss Procedure}

The CHL induction was a modified procedure from Tucci et al. (1999) (Figure 1A; Manno et al., 2020). Rats were anaesthetized 
with a cocktail of ketamine and xylazine (80-100 mg/kg: 5$10 \mathrm{mg} / \mathrm{kg}$ ) via intraperitoneal injection. A surgical field was setup and the head of the rat was aligned according to the prone position, closest to the experimenter. The helix of the rat ear was extended to cause the external auditory canal (i.e., ear canal and external auditory meatus) to become perpendicular to the surface of the tympanic membrane (TM). Micro-scissors (Micro spring scissors, RWD Life Science, and S11035-08) were introduced in the center of the auditory canal paying attention not to skim/nick the skin of the canal (Figure 1A). The scissors were introduced slowly, approximately $5 \mathrm{~mm}$ depth from the center of obscurity. The scissors were thrusted forward gently through the center of the TM. The thrusting elicited TM puncture (Figure 1A). With the micro-scissors in the appropriate position and correct depth, a "pop" sound was noticed when the micro-scissor tips puncture the TM displacing the malleus (Figure 1A). The popping sound was $\approx 20 \mathrm{~dB}$ SPL from background sound change as recorded by a high frequency microphone (M50, Earthworks, Chesterfield, MO, United States). After puncturing the TM, the micro-scissors were immediately opened and rotated three times to ensure displacement of the head of the malleus (Figure 1A). The microscissors were removed, and the rat placed under otoscope to visualize and ensure TM puncture (Otoscope mini 3000, HEINE, D-008.70.120M, and Standard LED otoscope). It is important to note no significant bleeding after the surgical procedure. Each rat was evaluated before and after CHL underneath otoscope for visualization and confirmation of normal TM and damaged TM after CHL induction. After CHL induction, the head of the malleus should be missing and not visible through the TM (Figure 1A). Anti-inflammatory drugs were supplied in the water for 1 week.

\section{Magnetic Resonance Imaging Acquisition}

Experiments were performed on a seven Tesla MRI scanner with a maximum gradient of $360 \mathrm{mT} / \mathrm{m}$ (70/16 PharmaScan, Bruker Biospin, Ettlingen, and Germany) using a transmitonly birdcage coil in combination with an actively decoupled receive-only surface coil (Figure 1A; Manno et al., 2019). The animals were initially anesthetized with $3 \%$ isoflurane. When sufficiently anesthetized, 1-2 drops of $2 \%$ lidocaine were applied to the cords to provide local anesthesia before the endotracheal intubation. The animals were mechanically ventilated at ate of 54-56 $\mathrm{min}^{-1}$ with $1-1.5 \%$ isoflurane in room-temperature air using a ventilator (TOPO, Kent Scientific Corp., Torrington, CT, United States). During MRI, the animals were placed on a plastic cradle with the head fixed with a tooth bar and plastic screws in the ear canals. Rectal temperature was maintained at $\sim 37.0^{\circ} \mathrm{C}$ using a water circulation system. Continuous physiological monitoring was performed using an MRI-compatible system (SA Instruments, Stony Brook, NY, United States). Vital signs were within normal physiological ranges (rectal temperature: $36.5-37.5^{\circ} \mathrm{C}$, heart rate: $350-420$ beat $/ \mathrm{min}$, breathing: $54-56$ breath/min, and oxygen saturation: N95\%) throughout the duration of the experiment (Chan et al., 2009; Lau et al., 2011; Cheung et al., 2012a; Zhou et al., 2014). Scout T2-weighted
(T2W) images were first acquired to position the subsequent images in a reproducible manner. The scan geometry was four $1.0 \mathrm{~mm}$ thick slices along the coronal view, with $0.1 \mathrm{~mm}$ interslice distance, positioned according to the rat brain atlas (Paxinos and Watson, 2004) such that the third slice was centered on the IC (Bregma $-8.6 \mathrm{~mm}$; Figure 1B).

\section{Diffusion Tensor Imaging Acquisition}

The DTI scan sequence was a spin-echo 4-shot echo planar imaging sequence with 30 diffusion gradient directions and $b$-value $=1,000 \mathrm{~s} / \mathrm{mm}^{2}$, and five images without diffusion sensitization ( $b=0.0 \mathrm{~ms} / \mu \mathrm{m}^{2}, \mathrm{~b} 0$ images) were acquired. Images with motion artifacts were discarded. The imaging parameters were: repetition time (TR)/echo time $(\mathrm{TE})=3,000 / 31.6 \mathrm{~ms}$, $\delta / \Delta=5 / 17 \mathrm{~ms}$, field of view $=3.2 \times 3.2 \mathrm{~cm}^{2}$, data matrix $=128$ $\times 128$, up-sampled data matrix $=256 \times 256$, cropped data matrix $=128 \times 128$, and number of excitements $=4$. Anatomical images were acquired with rapid acquisition with refocusing echoes (RARE). The imaging parameters were $\mathrm{RARE}$ factor $=8$, $\mathrm{TR} / \mathrm{TE}=4,200 / 32 \mathrm{~ms}$, field of view $=3.2 \times 3.2 \mathrm{~cm}^{2}$, data matrix $=256 \times 256$.

\section{Diffusion Tensor Imaging Analysis}

The diffusion-weighted images and b0 images were realigned and normalized to the b0 image of a template rat using SPM12 (Wellcome Trust Centre, Oxford, United Kingdom) and custom Matlab scripts (The MathWorks, Natick, MA, United States). DTI index maps were calculated by fitting the diffusion tensor model to the diffusion data at each voxel using DTIStudio v3.02 (Chan et al., 2009; Hui et al., 2010).

A map of color-coded directionality was generated, in which the color codes were for the principal eigenvector orientation while the contrast was weighted with FA. The following was used for depiction, white matter tracts running in the X-, Y-, and $\mathrm{Z}$ - directions were coded red, green, and blue, respectively (Pajevic and Pierpaoli, 1999). Therefore, the major eigenvector directional components for color-coded tractography (cFA) used the following naming convention: blue for superior-inferior, red for left-right, and green for anterior-posterior orientations (Jellison et al., 2004; Zhang et al., 2006). A voxel-wise $t$-test was performed on each DTI indices maps between the control and $\mathrm{CHL}$ rats to generate VBS. DTI images were spatially normalized by non-linear registration to the T2 structural image of the same rat. In brief, b0 and the T2 image were co-registered by a rigid transformation, followed by a two-dimensional affine transformation and a non-linear registration to improve the mapping between DTI and the structural image. The DTI index maps were calculated by fitting the diffusion tensor model to the diffusion data at each voxel using DTIStudio v3.02 (Johns Hopkins University, Baltimore, MD, and United States) as previously detailed (Chan et al., 2009; Hui et al., 2010; Abdoli et al., 2016). The normalized maps were smoothed with a $0.3 \mathrm{~mm}$ Gaussian kernel. The first and last slices were excluded in order to avoid truncation artifacts. The VBS clusters were considered significant at threshold $p<0.05$ and cluster size $>3$ voxels. The structures indicated by clusters were identified using the rat brain atlas (Paxinos and Watson, 2004; Figures 1B,C). 


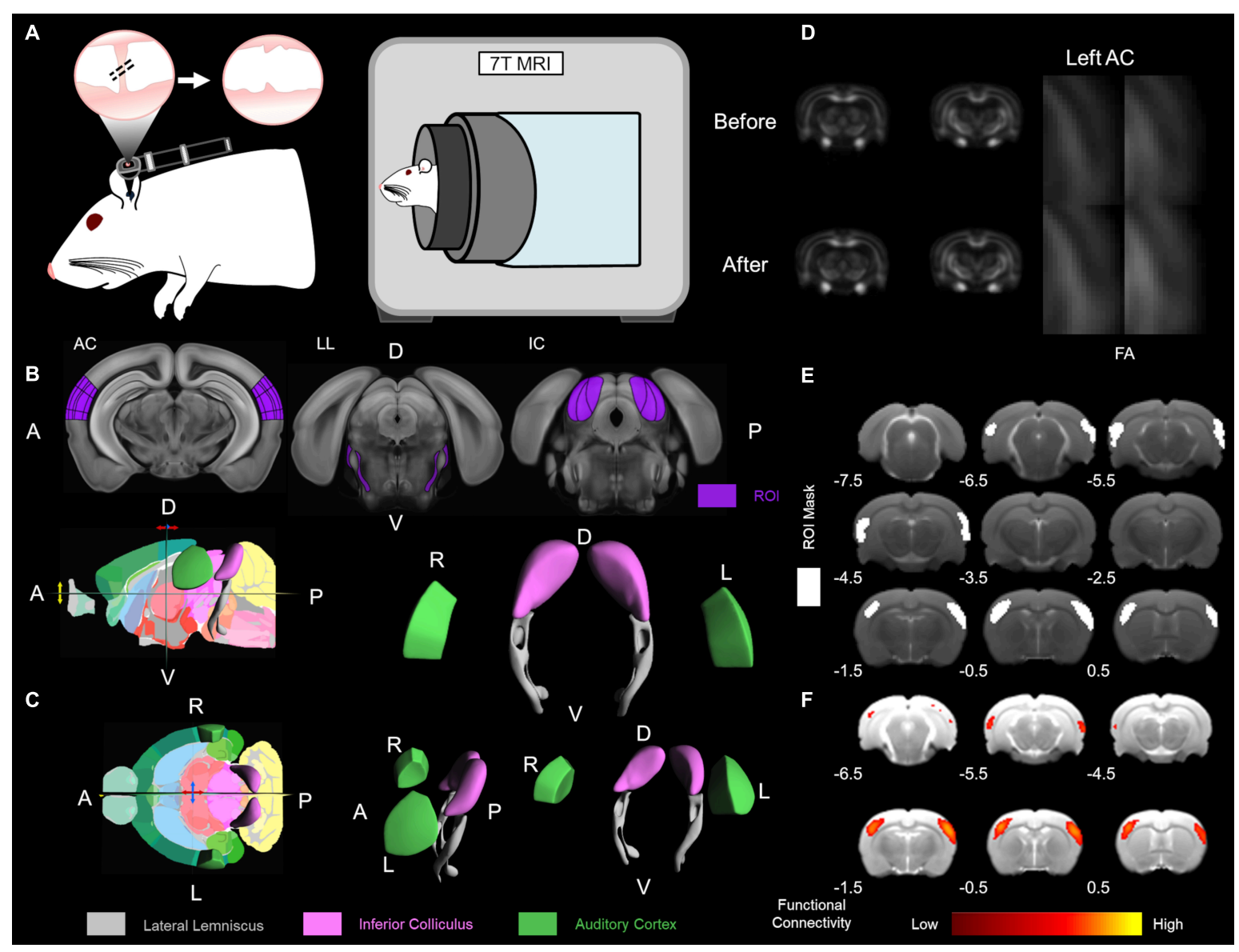

FIGURE 1 | Experimental design and data analysis. (A) The CHL surgery was conducted by tympanic membrane (TM) puncture and malleus displacement. A schematic of the rat ear with the otoscope highlighting the TM is depicted. Hash marks across the malleus indicate displacement and TM puncture with the serrated TM on the right. To the immediate right, the 7 Tesla magnetic resonance imaging (MRI) scanner for rodent structure-functional MRI (PharmaScan, Bruker Biospin). The Allen Mouse Brain Reference Atlas was used for visualization (Lein et al., 2007). (B) The Allen Mouse Brain Reference Atlas average template (Lein et al., 2007) with the region of interest (ROI), in dark purple, from left to right: AC 80 image, LL 96 image, and IC 101 image. (C) The Allen Mouse Brain Atlas 3D rendering a cutaway of the longitudinal/sagittal plane (top) and horizontal/transverse plane (bottom) with the 2D coronal reference atlas (Lein et al., 2007). The Allen Mouse Brain Atlas 3D volumetric rendering tilted in the horizontal plane rotated left $5^{\circ}$ (top), tilted in the horizontal plane rotated left $20^{\circ}$ (bottom), and tilted in the horizontal plane rotated left $90^{\circ}$ (bottom left), all in the $y$-axis translation. Volumetric ROI are color-coded lateral lemniscus in gray, inferior colliculus (IC) in light purple (lavender) and auditory cortex in green. R - right, L - left, D - dorsal, $\mathrm{V}$ - ventral, A - anterior, P - posterior. (D) Diffusion tensor imaging (DTI) fractional anisotropy (FA) of the before and 1 week after CHL timepoints. The left auditory cortex (AC) is highlighted in the zoomed insert. (E) The ROI for masks used for functional connectivity are delineated in white. Bregma coordinates (in $\mathrm{mm}$ ) are below the brain. (F) Functional connectivity maps using a color bar depicted from the correlation coefficient and z-score.

The region of interest (ROI) analysis (Figure 1B) was used to confirm that the structures identified by VBS were not significantly affected by normalization, registration, or other processing errors. Due to its confirmatory purpose, the ROI analysis was done in a conservative manner on unnormalized images. For each VBS identified structure, left and right hemisphere ROIs were drawn (Figure 1C). The ROIs were drawn such that they would be exclusive to the identified structure regardless of which rat they were applied to resulting in ROIs that were slightly smaller than the actual structure but still representative of the structure's general shape and size. All ROIs were of identical shape, size, and the left and right ROIs were always placed along the same horizontal axis. The only variation between the subjects was the distance between the right and left
ROI. The atlas was used to determine anatomical landmarks, such as the edge of the brain as the starting point of the lateral lemniscus tract, to serve as guidelines for placement of the ROIs. Once the ROIs were placed, FA, MD, $\mathrm{RD}\left(\lambda_{\perp}\right)$, and $\mathrm{AD}\left(\lambda_{/}\right)$ values were recorded for the left and right structures (Figure 1D). Values were recorded from the left and right sides to ensure that changes were occurring bilaterally.

Following ROI analysis, tractography was performed to ensure that ROIs drawn corresponded to the intended white matter structures. The ROI for the lateral lemniscus was placed on Bregma $-7.3,-8.3$, and $-9.3 \mathrm{~mm}$ and used to generate tracks. Tracking was performed on a voxel-by-voxel basis by continuously following the orientation of the first eigenvector of the tensor. All fiber bundles passing through the ROI on 
the second and third coronal slice which had a minimum FA threshold of 0.3 were followed until their FA was under 0.2 or the turning angle was greater than $60^{\circ}$. Using this methodology, the ventral aspect of the lateral lemniscus was delineated. The lateral lemniscus connects the IC to the lower brainstem superior olivary complex to the cochlear nucleus (Winer and Schreiner, 2005). The lateral lemniscus is a prominent pathway in GABAergic inhibition to the central nucleus of the IC (Li and Kelly, 1992; Zhang et al., 1998).

\section{Functional Magnetic Resonance Imaging Acquisition}

Similar to DTI, an anatomical image was first acquired using a $2 \mathrm{D}$ RARE sequence. The sequence parameters were RARE factor $=8$, field of view $=32 \times 32 \mathrm{~mm}^{2}$, data matrix $=256$ $\times 256, \mathrm{TR}=4200 \mathrm{~ms}$, and TE $=36 \mathrm{~ms}$. fMRI images were acquired with a gradient echo echo planar imaging (GE-EPI) sequence with the following parameters: field of view $=32 \times 32$ $\mathrm{mm}^{2}$, data matrix $=64 \times 64, \mathrm{TR}=1,000 \mathrm{~ms}, \mathrm{TE}=20 \mathrm{~ms}$, and 400 acquisitions. The EPI scan geometry was imported from the anatomical scan geometry. Twelve EPI scans were performed.

\section{Reduced Resting-State fMRI Analyses}

For each rsfMRI session, all images were first corrected for slice timing differences and then realigned to the first image of the series using SPM12 (Wellcome Department of Imaging Neuroscience, University College, London). A voxelwise linear detrending with least-squares estimation was performed temporally to eliminate the baseline drift caused by physiological noise and system instability. No spatial smoothing was performed while a temporal band-pass filtering $(0.005-0.1 \mathrm{~Hz})$ was applied. The first 15 image volumes and last 15 image volumes of each session were discarded to eliminate possible non-equilibrium effects. Finally, highresolution anatomical images from individual animals were coregistered to a custom-made brain template acquired from a separate age-matched rat with the same settings with a $3 \mathrm{D}$ rigid-body transformation and the transforming matrix was then applied to the respective rsfMRI data (Chan et al., 2015, 2017). To determine functional connectivity changes before and after $\mathrm{CHL}$, seed-based analysis (SBA) and independent component analysis (ICA) were performed.

For SBA, two $3 \times 3$ voxel regions were chosen as the ipsilateral and contralateral seed, respectively, in the primary AC, primary somatosensory cortex (S1) and the IC. Regionally averaged time course from the voxels within each seed served as the respective reference time course. Pearson's correlation coefficients were calculated between the reference time course and the time course of every other voxel to generate two rsfMRI connectivity maps for each region. A $3 \times 3$-voxel region on the contralateral side of the seed was defined as the ROI (Figure 1E). Interhemispheric functional connectivity for each region was then quantified by averaging the correlation coefficient value of the corresponding ipsilateral and contralateral ROI (Chan et al., 2015, 2017; Figure 1F).
For ICA, coregistered rsfMRI data was analyzed with the GIFT v4.0b Toolbox. The Infomax algorithm was used and group-level ICA was performed on all rsfMRI data at the same time point. The group-level spatial ICA maps of independent resting-state networks were scaled to z-scores with a threshold of $z>2.5$ (corresponding to a significance level of $p<0.01$ ). The ICA maps were then visually inspected and labeled based on the spatial patterns in reference to known anatomical and functional locations. AC and S1 resting-state networks were further quantified. ROIs covering the bilateral functional areas were defined according to the resting-state networks obtained (Figure 1E). Mean z-scores were obtained by averaging the values within the ROIs (Chan et al., 2015; Figure 1F).

\section{RESULTS}

\section{Structural Alterations Underlying CHL Assessed by VBS of DTI Images \\ (Figure 2)}

Voxel-based statistics were used to visualize areas altered 1 week after CHL (Figure 2). The VBS was mapped as a function of $t$-value change. The maximum point of significance for FA was $t=3.170$ and for $\mathrm{AD}$ was $t=2.729$. Clusters of voxels with significant differences were observed bilaterally in the AC. The visualization was done for Bregma -6.40 and $-5.3 \mathrm{~mm}$. The VBS analysis was followed by a DTI ROI analysis on the AC for determining $\mathrm{FA}, \mathrm{MD}, \mathrm{RD}\left(\lambda_{\perp}\right)$, and $\mathrm{AD}\left(\lambda_{/} /\right)$.

\section{Structural Alterations Underlying $\mathrm{CHL}$ Assessed by DTI ROI Analysis (Figure 3)}

The primary $\mathrm{AC}$ was delineated, first by each individual rat, and then group-averaged (Figures $\mathbf{3 A}, \mathbf{B}$ ). The panel is segregated into before and after CHL for the left and right hemispheres on the coronal section Bregma $-3.48 \mathrm{~mm}$. The FA and AD for the primary AC was significantly decreased after CHL (Figure 3C). The mean FA for AC after CHL was significantly decreased compared to before (before FA $0.1612 \pm 0.0020$ and after FA $0.1526 \pm 0.0021 ; T$-test: $t=2.769, \mathrm{df}=12, p=0.0143)$. The mean $\mathrm{AD}$ for $\mathrm{AC}$ after $\mathrm{CHL}$ was significantly decreased compared to before (before $\mathrm{AD} 0.8760 \pm 0.0064$ and after $\mathrm{AD} 0.8513 \pm 0.0077$; $T$-test: $t=2.474, \mathrm{df}=15$, and $p=0.0258)$. No change was observed for the primary somatosensory cortex.

\section{Structural Alterations Underlying CHL Assessed by Tractography (Figure 4)}

Tractography was used to delineate the ventral aspect of lateral lemniscus, a prominent tract in the rat (Winer and Schreiner, 2005; Figure 4A). The color map shows the primary eigenvector in the region of the ventral aspect of the lateral lemniscus is along the posterior-anterior direction and traversing upward in the ventral-dorsal direction (Figure 4B - ROI). The tractography panel (Figure 4B) demonstrates the fibers originate from a ventral position and course upward dorsally in a bilaterally symmetrical fashion. Analysis of fiber number, fiber length and fiber density using tractography found CHL elicited a significant 


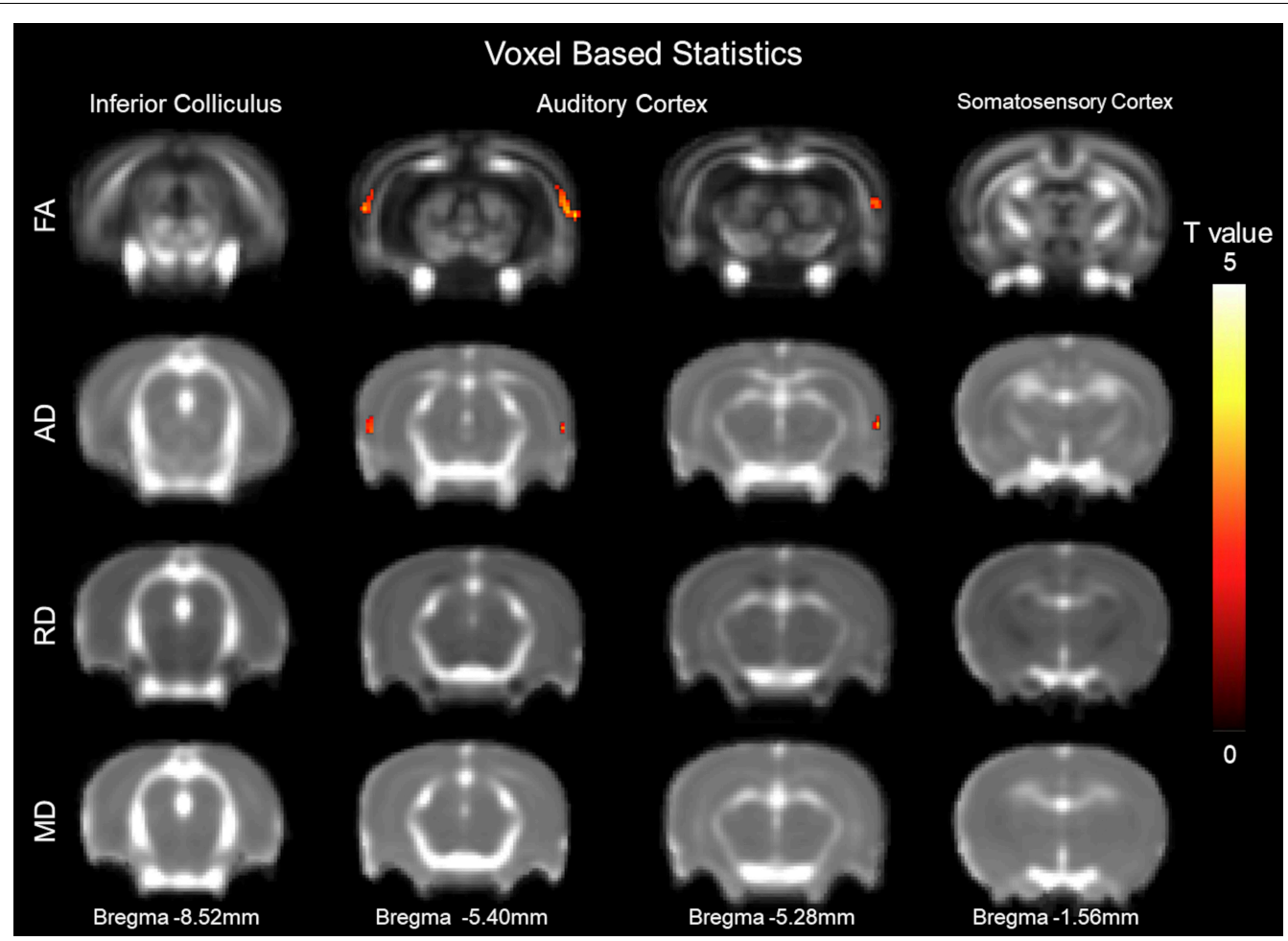

FIGURE 2 | Structural alterations underlying conductive hearing loss assessed by DTI voxel-based statistics. The y-axis representing rows are fractional anisotropy $(F A)$, axial diffusivity $(A D, \lambda / /)$, radial diffusivity $\left(\mathrm{RD}, \lambda_{\perp}\right)$, and mean diffusivity (MD). Columns represent different Bregma locations $-8.6,-6.40,-5.3$, and -2.0 mm. Color bars represent positive $t$ value differences between before and after $\mathrm{CHL}$ (warm colors from 0 to 5 ). The maximum point of significance for $\mathrm{FA}$ was $t=3.170$, and for $\mathrm{AD}$ was $t=2.729$.

reduction in tract length and fiber density $(p<0.05$; Figure 4C). The mean fiber number for the lateral lemniscus after CHL was insignificantly different for CHL (before 71.07 $\pm 5.684, N=14$; after $64.39 \pm 4.381, N=18$; $T$ - test: $t=0.9474$, df $=30$, and $p=0.3510)$. The mean fiber length for the lateral lemniscus after CHL was significantly decreased compared to before (before $2.891 \mathrm{~mm} \pm 0.1076 \mathrm{~mm}, N=14$; after $2.590 \mathrm{~mm} \pm 0.06218 \mathrm{~mm}$, $N=18$; $T$-test: $t=2.548, \mathrm{df}=30$, and $p=0.0162$ ). The mean fiber density for the lateral lemniscus after CHL was significantly decreased compared to before (before $3.414 \mathrm{~mm}^{3} \pm 0.1422 \mathrm{~mm}^{3}$, $N=14$; after $2.923 \mathrm{~mm}^{3} \pm 0.1438 \mathrm{~mm}^{3}, N=18$; $T$-test: $t=2.388$, $\mathrm{df}=30$, and $p=0.0234$ ).

\section{Functional Connectivity Alterations After $\mathrm{CHL}$ as Assessed by rsfMRI (Figure 5)}

Figure 5A shows ROIs delineating the seeds from SBA and the masks from ICA. The mean correlation coefficient maps (Figure 5) demonstrate the presence of the bilateral auditory and somatosensory cortices, and IC rsfMRI networks before and after CHL. For rsfMRI, 8-10 rats passed the quality control for SBA $(\mathrm{df}=20)$ and ICA $(\mathrm{df}=18)$ assessment. The SBA correlation coefficient for rsfMRI connectivity was significantly decreased in the AC by $42.27 \%$ (Bonferroni's post hoc test, $p<0.01$ ) after CHL (before $0.3106 \pm 0.9178 \mathrm{CI}: 0.2523-0.3689$ and after $0.1793 \pm 0.1094 \mathrm{CI}: 0.1098-0.2488, t=3.185, \mathrm{df}=22$, and $p=0.0043)$. Note that the bilateral somatosensory network revealed no significant connectivity strength changes between the before and after CHL timepoint (before: $0.2728 \pm 0.0890$; CI: $0.2091-0.3365$, and after: $0.2749 \pm 0.1043$; CI: $0.2003-$ $0.3495, t=0.04890, \mathrm{df}=18, p=0.9615$, difference between means: $0.21 \pm 4.347$ ). The SBA correlation coefficient for rsfMRI connectivity was significantly decreased in the IC by 42.50\% (Bonferroni's post hoc test, $p<0.01$ ) after CHL (before: $0.4576 \pm 0.1867 \mathrm{CI}: 0.3241-0.5912$ and after: $0.2631 \pm 0.09272$ CI: $0.1967-0.3294, t=2.952, \mathrm{df}=18$, and $p=0.0085)$. The SBA revealed a bilateral decrease in rsfMRI connectivity in the AC and IC after CHL (Figure 5B) indicating these ROI became less functionally connected.

The z-score maps (Figure 5C) demonstrate the presence of the bilateral auditory and somatosensory cortex rsfMRI networks before and after CHL. The ICA z-score for rsfMRI connectivity was decreased in the AC by $27.11 \%$ after $\mathrm{CHL}$ (before: $2.058 \pm 0.5206 \mathrm{CI}: 1.512-2.605$ and after: $1.500 \pm 0.3815$ CI: $1.100-1.901) t=2.117, \mathrm{df}=10$, and $p=0.063$. Note that bilateral somatosensory network revealed no significant connectivity strength changes between the before and after CHL timepoint (before: $3.155 \pm 0.6749 \mathrm{CI}: 2.727-3.584$ and after: $3.037 \pm 0.7700 \mathrm{CI}: 2.547-3.526) t=0.4020$, df $=22$, $p=0.6915$, difference between means: $11.88 \pm 29.56)$. The ICA revealed a bilateral decrease in rsfMRI connectivity in the AC after CHL (Figure 5C), indicating the ROI became less functionally connected. 


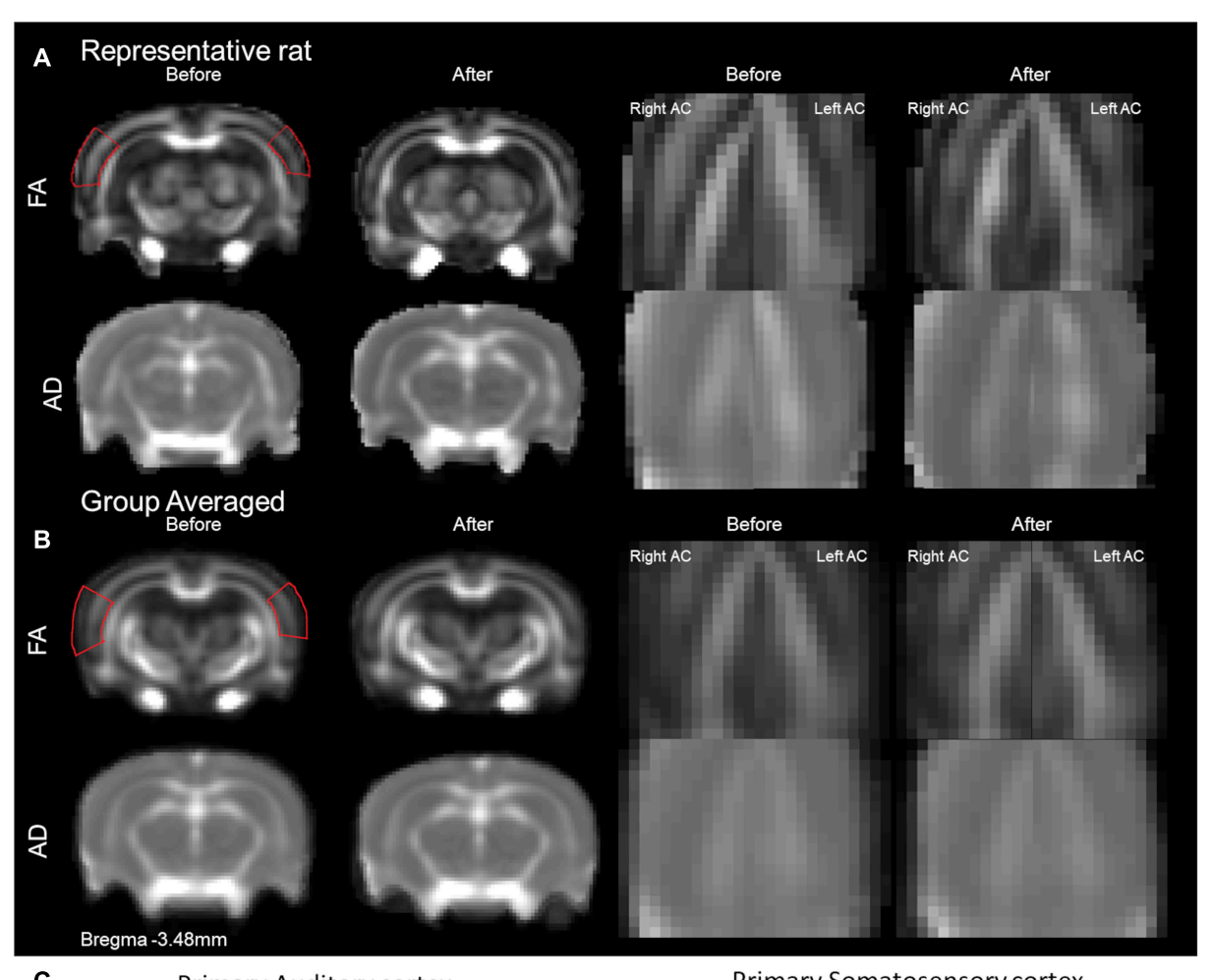

C Primary Auditory cortex

Primary Somatosensory cortex
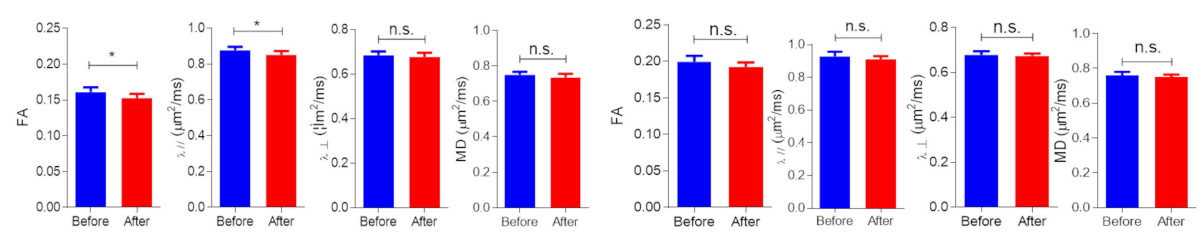

FIGURE 3 | Structural alterations underlying conductive hearing loss assessed by DTI ROls. The panel is segregated into before and after CHL for Bregma $-3.48 \mathrm{~mm}$. (A) Representative rat FA maps (first row of brains from left) and AD maps (second row of brains from left) from individual $\mathrm{CHL}$ rats. (B) Group-averaged FA (first row of brains from left) and $A D$ (second row of brains from left) maps for $\mathrm{CHL}$. Note the red outline for FA represents the approximate ROI for measures. (C) Bar plots of DTI measures for the primary auditory cortex and somatosensory cortex: FA, MD, RD ( $\left.\lambda_{\perp}\right)$, and AD ( $\left.\lambda / /\right)$ for before (blue) and after (red) CHL with standard deviation. The mean FA and AD after $\mathrm{CHL}$ were significantly decreased compared to before, $T$-test: $t=2.769, \mathrm{df}=12, p=0.0143$ and $t=2.474, \mathrm{df}=15$, and $p=0.0258$, respectively. The asterisk $\left(^{*}\right)$ indicates significant $p<0.05$.

\section{DISCUSSION}

Bilateral CHL produces air-conducted sound attenuation (indicating no cochlear damage: Tucci et al., 1999), resulting in task-specific behavioral threshold increases of $\approx 35 \mathrm{~dB}$ and neural auditory brainstem response threshold increases of $\approx 40 \mathrm{~dB}$ for sinusoidally AM stimuli (Rosen et al., 2012). The auditory system is greatly impacted due to $\mathrm{CHL}$, yet investigations into structural-functional correlations in hearing loss are lacking. Here we demonstrated CHL results in structural and functional alterations in the IC and AC. Structurally, FA and axial diffusivity alterations are found in the $\mathrm{AC}$ and tractography revealed the lateral lemniscus tract leading to the inferior cortex bilaterally was reduced. Functionally after CHL, SBA revealed functional connectivity decreased in the AC and IC, while ICA revealed functional connectivity decreased in the AC, while both these assessments found no change in functional connectivity in the somatosensory cortex. The present results indicate structural alterations from short term hearing loss are associated with altered functional connectivity.

Rodent models recapitulate some of the main features of the neuropathology of human hearing loss. The research in rats and mice range from brief noise exposure to chronic conditions. In rats, long-term passive noise exposure has revealed greater FA in the pyramidal tract and decreased FA in the tectospinal tract and trigeminothalamic tract (Abdoli et al., 2016). These tracts are involved in voluntary control of the body and limbs, transmit noxious stimuli from the face and motor pathway which reflexively controls the muscles of the head for visual stimuli, for the pyramidal, trigeminothalamic, and tectospinal tracts, respectively (Abdoli et al., 2016). In the rat, these tracts possibly help to orient the rat to an alarm such as the acoustic startle reflex; thus, adjusting body coordination to sound stimuli. This is interesting as a previous experiment has demonstrated 


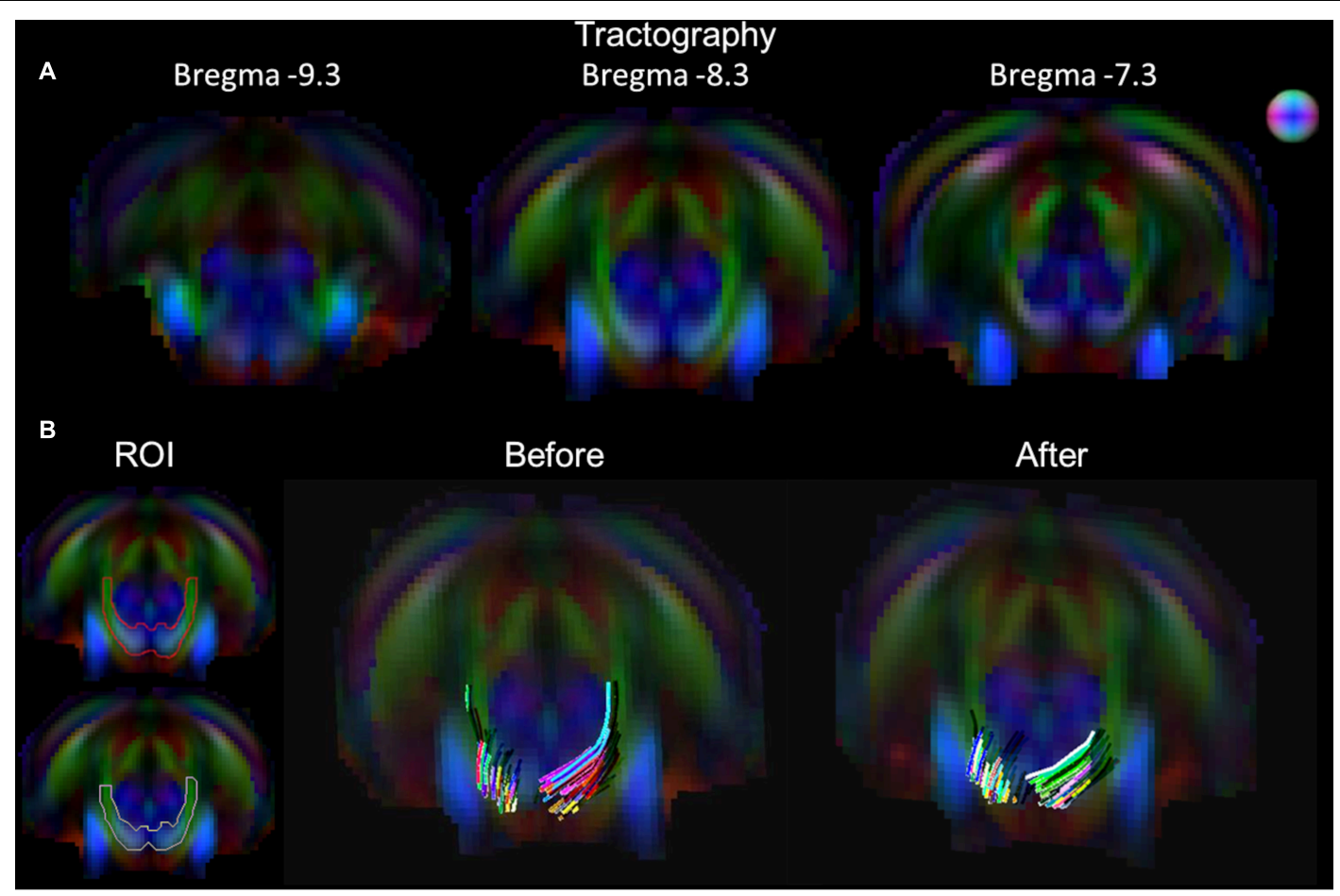

C

Tract statistics
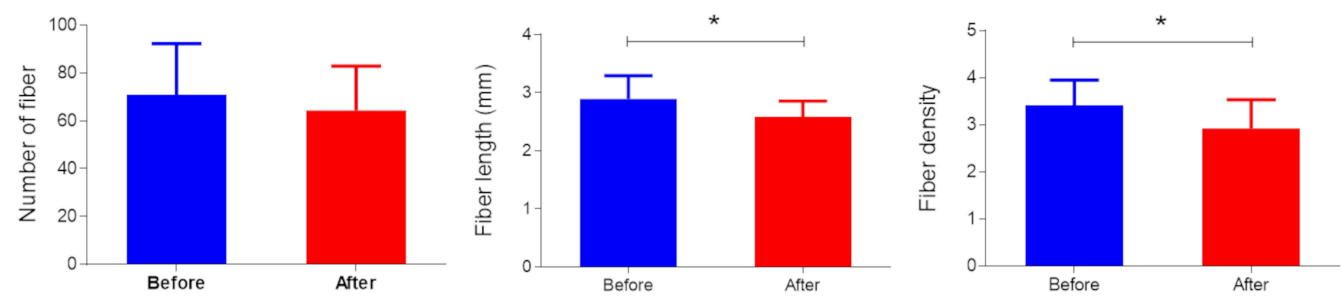

FIGURE 4 | Tract alterations underlying conductive hearing loss assessed by tractography. (A) Tractography was used to delineate the ventral aspect of lateral lemniscus on Bregma $-7.5,-8.6$, and $-9.7 \mathrm{~mm}$. The color map shows the primary eigenvector in the region of the ventral lateral lemniscus traversing upward in the ventral-dorsal direction. (B) The tractography panel $\mathrm{ROI}$ demonstrates the fibers originate from a ventral position and course upward dorsally in a bilaterally symmetrical fashion. (C) Bar plots of tract metrics in the ROI for the lateral lemniscus. The mean fiber number was insignificantly different after $\mathrm{CHL}$; however, the mean fiber length and mean fiber density for the lateral lemniscus after $\mathrm{CHL}$ was significantly decreased compared to before $(t$-test: $t=2.548, \mathrm{df}=30, p=0.0162$ and $t=2.388$, $d f=30$, and $p=0.0234$, respectively).

reflex inhibition produced by brief noise exposure (Cory and Ison, 1979; Wu et al., 1984), indicating in the rat a priming or sensitization effect. It is interesting to note, an increased sensitivity to continuous noise results in suppression in the acoustic startle response (ASR) in rats (Rybalko et al., 2019). In the present study, we demonstrated an approximate $\approx 35 \mathrm{~dB}$ attenuation (Sohmer et al., 1991; Sohmer and Friedman, 1992; Rosen et al., 2012; Ye et al., 2021), resulted in a decrease in fiber length and fiber density in the lateral lemniscus and decrease in $\mathrm{FA}$ and $\mathrm{AD}$ for $\mathrm{AC}$ after 1-week of hearing loss in postnatal day 60 rats. The current study performed hearing loss at postnatal day 60 (considered young adults, McCutcheon and Marinelli, 2009) and examined changes over a brief period of time (1 week). A study measuring the impact of conductive hearing loss induced developmentally (postnatal day 10) found perceptual deficits later on in adulthood (postnatal day 70). Juvenile onset hearing loss
$(89 \mathrm{~Hz})$ had greater frequency modulation detection thresholds $(89 \mathrm{~Hz})$ than adult onset hearing loss $(64 \mathrm{~Hz}$; Buran et al., 2014). The study indicated decreased auditory stimulation at an early age had shallower psychometric functions likely leading to altered brain development. In the current study, we demonstrate what the altered brain looks like due to impoverished hearing in adults, possibly more affected if induced during the development, altricial period. Interestingly, in salicylate- and noise-induced tinnitus model, the IC exhibited supernormal uptake manganeseenhanced MRI implicating its role in tinnitus-related neuronal activity (Holt et al., 2010). The IC is a prominent site of brain stem convergence and multisensory integration in the auditory pathway of the rat (Winer and Schreiner, 2005). The results support the conclusion that the IC (Figure 5C) and tracts leading to this midbrain structure such as the lateral lemniscus (Figure 4B) are intimately involved in the first 

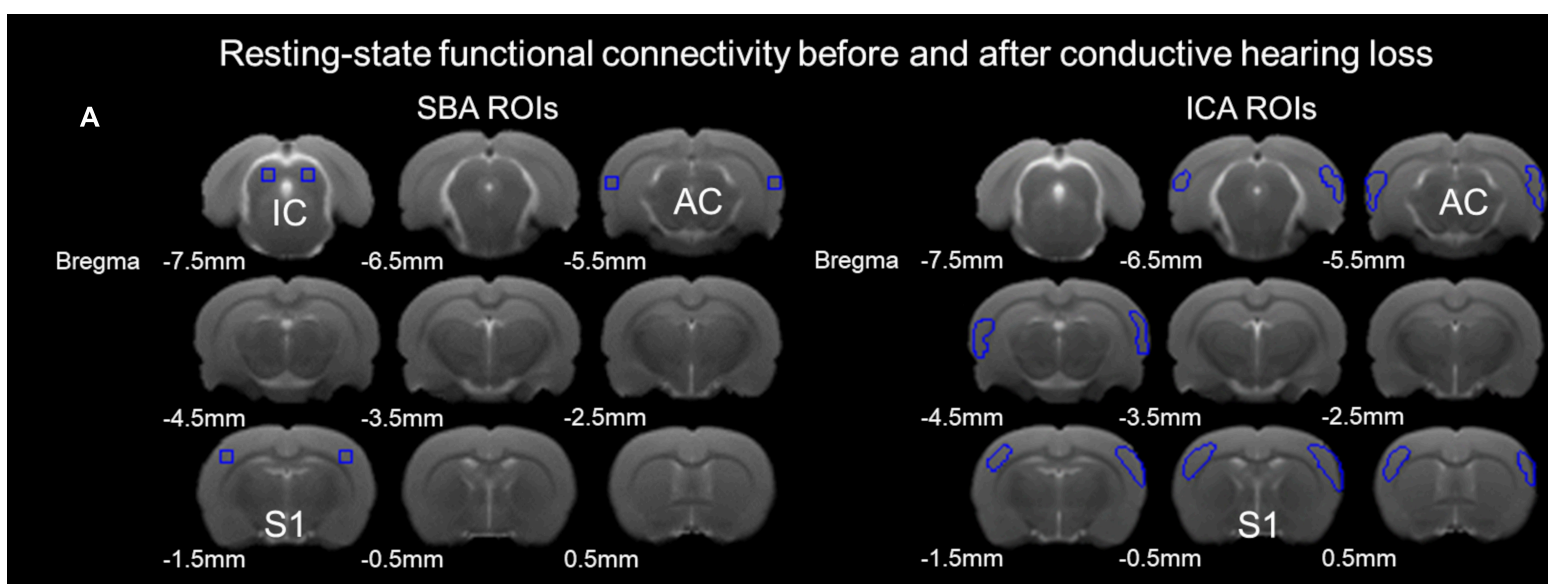

B

Seed based analysis functional connectivity maps

Seed based an
Ipsilateral seed
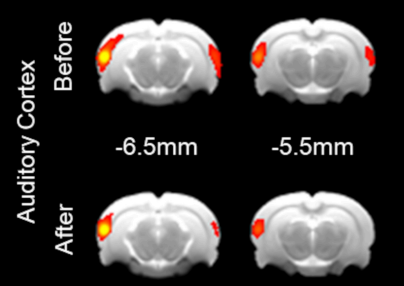

Ipsilateral seed
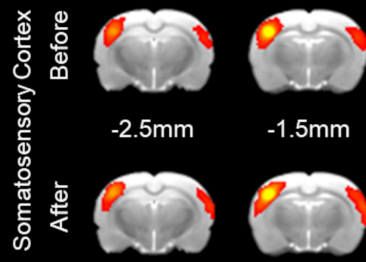

Ipsilateral seed
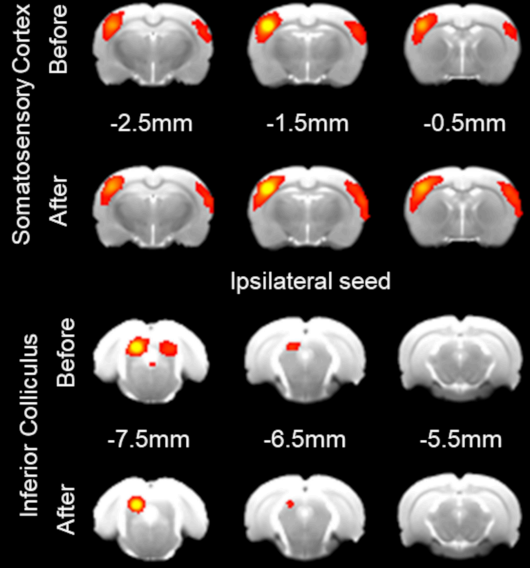

Contralateral seed

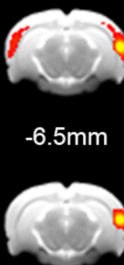

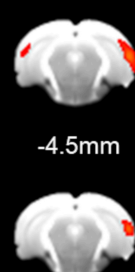

Contralateral seed
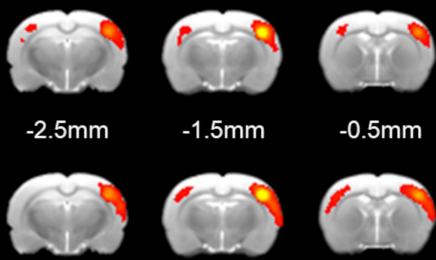

Contralateral seed

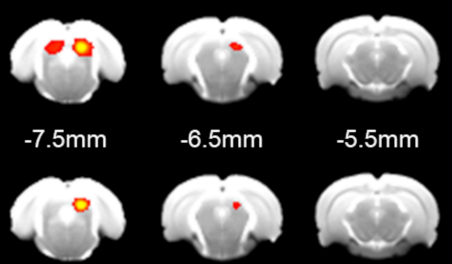

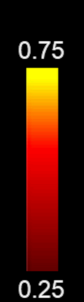

0.25

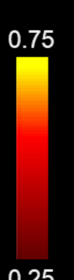

0.25

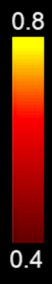

C

Independent component analysis functional connectivity maps
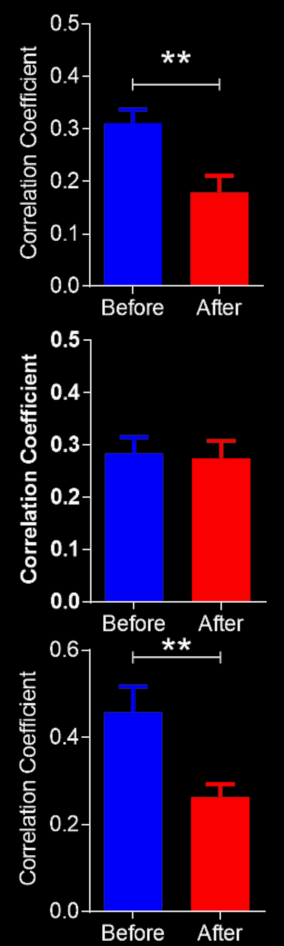

Primary Auditory Network

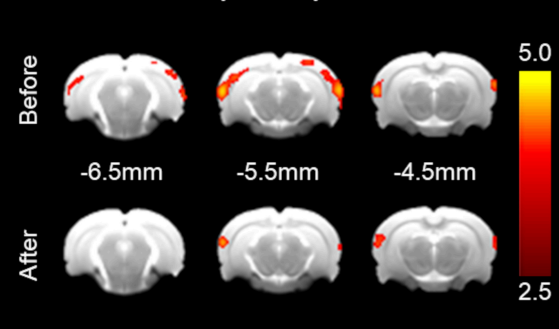

Primary Somatosensory Network
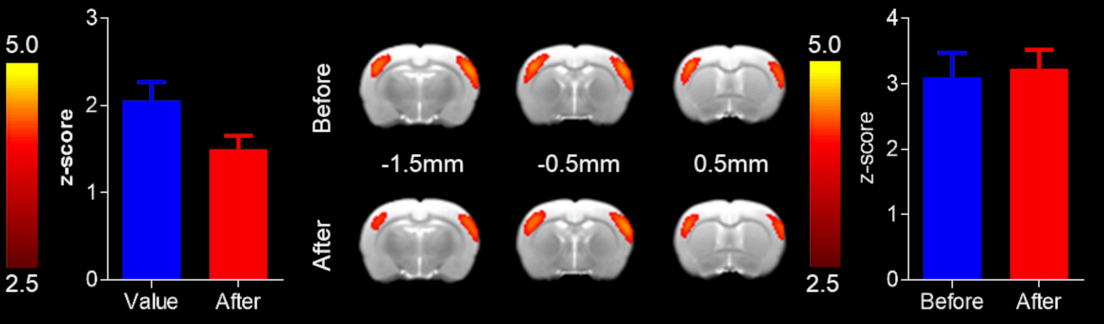

FIGURE 5 | Functional connectivity alterations resulting from conductive hearing loss as assessed by rsfMRI. (A) Seeds used for SBA and masks for ICA were used to delineate ROI for the bilateral auditory cortex, somatosensory cortex, and IC rsfMRI networks. (B) The SBA rsfMRI connectivity was significantly decreased in the auditory cortex and IC after CHL $(p<0.01)$, however, remaining unchanged in the somatosensory cortex. The bar plots indicate the correlation coefficient before (blue) and after (red) $\mathrm{CHL}$, demonstrating a decrease of $42.27 \%$ and $42.50 \%$ for the auditory cortex and IC, respectively. (C) The ICA rsfMRI connectivity was decreased in the auditory cortex after CHL, and was unchanged in the somatosensory cortex. The bar plots indicate the $\mathrm{z}$-score before (blue) and after (red) $\mathrm{CHL}$, demonstrating a decrease of $27.11 \%$ for the auditory cortex. Both SBA and ICA demonstrate rsfMRI networks decrease after CHL. 
processes of structural-functional reorganization due to CHL. Its likely the cochlear nucleus, lateral lemniscus and IC, in addition to other midbrain structures (e.g., superior olivary complex; Malmierca, 2003; Osen et al., 2019), are affected in a reentrant manner, first with lack of external stimuli (bottom-up) and second with the release of top-down processing (Caras and Sanes, 2017). Future experiments should attempt to distinguish the relative contributions to both processing pathways (topdown/bottom-up). Further, using a salicylate-induced model of hyperacusis, we found AC tone-evoked BOLD fMRI signals during salicylate treatment were consistently larger than saline (indicating evidence of enhanced central gain; Wong et al., 2020). Interestingly during this model of hyperacusis, the lateral lemniscus and IC BOLD response obtained during salicylate treatment were similar to saline control values (Wong et al., 2020), indicating a top-down first contribution. Acute noise paradigms have used fMRI and implanted electrodes to mixed results. Acute noise exposure reduced midbrain IC sound-evoked responses as detected using fMRI by as early as 1 week (Yang et al., 2018). Using implanted electrodes in an acoustic startlereflex paradigm, a decrease in sound evoked potential was seen in IC; however, an increase was observed in AC, after noise exposure (Sun et al., 2012). In the present study with rats, we have demonstrated short-term early affect to auditory structures due to CHL. Future studies should establish the interrelations between these structures over the longitudinal course of hearing loss.

In humans, we have recently assessed the structural foundations of hearing loss across the lifespan (Manno et al., 2021). Our systematic review and meta-analysis revealed $n=72$ studies with structural alterations measured by MRI (bilateral $=64$, unilateral $=8$ ). The studies contained more than 66,545 variable datapoint metrics categorizing hearing loss into congenital and acquired cases from mild to profound impact $(n=7445)$ and control cases $(n=2924)$. This large longitudinal study found the endophenotype of hearing loss is heterogeneous, determined by gray matter alterations, and results in a widespread impact to the brain of pediatric, adult and aged-adult populations whether the etiology is congenital or acquired. The most important finding of our study was hearing loss is a unique sensory disorder, resembling a diffuse brain disorder, impacting regions and tracts differently depending on auditory input and compensatory mechanisms. In humans, several fMRI studies have documented the functional effects to hearing loss (Schmidt et al., 2013; Liu et al., 2015; Puschmann and Thiel, 2017; Xia et al., 2017; Fitzhugh et al., 2019; Luan et al., 2019, 2020; Wang et al., 2019; Wolak et al., 2019; Xu et al., 2019), although primarily congenital bilateral hearing loss. Nevertheless, reduced rsfMRI connectivity has been found to accompany hearing loss (as would be expected to AC, when sound stimuli is reduced) based on tone stimulation paradigms (Scheffler et al., 1998; Propst et al., 2010; Tibbetts et al., 2011). The functional MRI studies complement our structural MRI study indicating changes to structural-functional coupled relationships can be attributed to widespread brain regions due to the impact of hearing loss.
There are several limitations in this assessment of CHL structure-functional coupling. The sample size in the current study is relatively low ( $N=9-12$, separated by 1 -week), which may have contributed to the low signal to noise ratio in the rsfMRI data quality. Future studies should consider increasing the sample size to address data quality concerns as well as imaging multiple timepoints to develop a longitudinal assessment of CHL. Furthermore, our DTI processing pipeline (Manno et al., 2019) may not have been sensitive enough to detect all the alterations seen in CHL during this short-term period. Optimization and improvement in the pipeline and scanning protocol for subsequent CHL studies might allow more accurate detection of structural-functional alterations for a variety of structures impacted due to hearing loss.

\section{DATA AVAILABILITY STATEMENT}

The datasets presented in this study can be found in online repositories. The names of the repository/repositories and accession number(s) can be found below: https://francismanno. github.io/fmanno/.

\section{ETHICS STATEMENT}

The animal study was reviewed and approved by the Animal Research Ethics Sub-Committee of the City University of Hong Kong.

\section{AUTHOR CONTRIBUTIONS}

CL, YF, and FM organized the CHL project at The City University of Hong Kong. FM, ZA, RK, YF, and CL designed research. FM, $\mathrm{ZA}$, and $\mathrm{YX}$ performed the research and data curation. FM, ZA, and RK analyzed the data. FM, ZA, RK, EW, JH, YF, and CL wrote and edited the manuscript. YF and CL acquired funding.

\section{FUNDING}

FM was supported by the Research Grants Council of Hong Kong SAR, China HKPFS \# PF16-07754. RK was supported by the Medical Scientist Training Program NIH grant T32 GM07170. YF was supported by the General Program of National Natural Science Foundation of China Grant Nos. 61671228, 61728107, and 81871349 and the Technology R\&D Program of Guangdong (Grant Nos. 2017B090912006 and 2018B030333001). CL was supported by the Early Career Scheme, Research Grants Council of Hong Kong project \#21201217.

\section{ACKNOWLEDGMENTS}

All authors would like to thank Dr. Sinai H. C. Manno for critical comments and assistance in preparing the manuscript and Chan Chi Ming (Sam) for his technical assistance. 


\section{REFERENCES}

Abdoli, S., Ho, L. C., Zhang, J. W., Dong, C. M., Lau, C., and Wu, E. X. (2016). Diffusion tensor imaging reveals changes in the adult rat brain following longterm and passive moderate acoustic exposure. J. Acoust. Soc. Am. 140:4540. doi: $10.1121 / 1.4972300$

Buran, B. N., Sarro, E. C., Manno, F. A., Kang, R., Caras, M. L., and Sanes, D. H. (2014). A sensitive period for the impact of hearing loss on auditory perception. J. Neurosci. 34, 2276-2284. doi: 10.1523/jneurosci.0647-13.2014

Caras, M. L., and Sanes, D. H. (2017). Top-down modulation of sensory cortex gates perceptual learning. Proc. Natl. Acad. Sci. U.S.A. 114, 9972-9977. doi: 10.1073/pnas.1712305114

Chan, K. C., Khong, P. L., Cheung, M. M., Wang, S., Cai, K. X., and Wu, E. X. (2009). MRI of latemicrostructural and metabolic alterations in radiationinduced brain injuries. J. Magn. Reson. Imaging 29, 1013-1020. doi: 10.1002/ jmri.21736

Chan, R. W., Ho, L. C., Zhou, I. Y., Gao, P. P., Chan, K. C., and Wu, E. X. (2015). Structural and functional brain remodeling during pregnancy with diffusion tensor MRI and resting-state functional MRI. PLoS One 10:e0144328. doi: 10.1371/journal.pone. 0144328

Chan, R. W., Leong, A. T. L., Ho, L. C., Gao, P. P., Wong, E. C., Dong, C. M., et al. (2017). Low-frequency hippocampal-cortical activity drives brain-wide resting-state functional MRI connectivity. Proc. Natl. Acad. Sci. U.S.A. 114, E6972-E6981.

Cheung, M. M., Lau, C., Cheng, J. S., Zhang, J. W., Zhou, I. Y., Chan, K. C., et al. (2012a). BOLD fMRI investigation of the rat auditory pathway and tonotopic organization. Neuroimage 60, 1205-1211. doi: 10.1016/j.neuroimage.2012.0 1.087

Cheung, M. M., Lau, C., Zhou, I. Y., Chan, K. C., Zhang, J. W., Fan, S. J., et al. (2012b). High fidelity tonotopic mapping using swept source functional magnetic resonance imaging. Neuroimage 61, 978-986. doi: 10.1016/j. neuroimage.2012.03.031

Cory, R. N., and Ison, J. R. (1979). Persistent effect of noise on the acoustic startle reflex in the rat. Anim. Learn. Behav. 7, 367-371. doi: 10.3758/BF03209686

Dong, C. M., Leong, A. T. L., Manno, F. A. M., Lau, C., Ho, L. C., Chan, R. W., et al. (2018). Functional MRI investigation of audiovisual interactions in auditory midbrain. Conf. Proc. IEEE Eng. Med. Biol. Soc. 2018, 5527-5530. doi: 10.1109/ embc.2018.8513629

Fitzhugh, M. C., Hemesath, A., Schaefer, S. Y., Baxter, L. C., and Rogalsky, C. (2019). Functional connectivity of Heschl's Gyrus associated with age-related hearing loss: a resting-state fMRI study. Front. Psychol. 10:2485. doi: 10.3389/ fpsyg.2019.02485

Holt, A. G., Bissig, D., Mirza, N., Rajah, G., and Berkowitz, B. (2010). Evidence of key tinnitus-related brain regions documented by a unique combination of manganese-enhanced MRI and acoustic startle reflex testing. PLoS One 5:e14260. doi: 10.1371/journal.pone.0014260

Hui, E. S., Cheung, M. M., Chan, K. C., and Wu, E. X. (2010). Bvalue dependence of DTI quantitation and sensitivity in detecting neural tissue changes. Neuroimage 49, 2366-2374. doi: 10.1016/j.neuroimage.2009.1 0.022

Jellison, B. J., Field, A. S., Medow, J., Lazar, M., Salamat, M. S., and Alexander, A. L. (2004). Diffusion tensor imaging of cerebral white matter: a pictorial review of physics, fiber tract anatomy, and tumor imaging patterns. AJNR Am. J. Neuroradiol. 25:356-369.

Kotak, V. C., Fujisawa, S., Lee, F. A., Karthikeyan, O., Aoki, C., and Sanes, D. H. (2005). Hearing loss raises excitability in the auditory cortex. J. Neurosci. 25, 3908-3918. doi: 10.1523/jneurosci.5169-04.2005

Kotak, V. C., and Sanes, D. H. (1997). Deafferentation weakens excitatory synapses in the developing central auditory system. Eur. J. Neurosci. 9, 2340-2347. doi: 10.1111/j.1460-9568.1997.tb01651.x

Kotak, V. C., Takesian, A. E., MacKenzie, P. C., and Sanes, D. H. (2013). Rescue of inhibitory synapse strength following developmental hearing loss. PLoS One 8:e53438. doi: 10.1371/journal.pone.0053438

Kotak, V. C., Takesian, A. E., and Sanes, D. H. (2008). Hearing loss prevents the maturation of GABAergic transmission in the auditory cortex. Cereb. Cortex 18, 2098-2108. doi: 10.1093/cercor/bhm233

Lau, C., Manno, F. A. M., Dong, C. M., Chan, K. C., and Wu, E. D. (2018). Auditory-visual convergence at the superior colliculus in rat using functional
MRI. Conf. Proc. IEEE Eng. Med. Biol. Soc. 2018, 5531-5536. doi: 10.1109/embc. 2018.8513633

Lau, C., Pienkowski, M., Zhang, J. W., McPherson, B., and Wu, E. X. (2015a). Chronic exposure to broadband noise at moderate sound pressure levels spatially shifts tone-evoked responses in the rat auditory midbrain. Neuroimage 122, 44-51. doi: 10.1016/j.neuroimage.2015.07.065

Lau, C., Zhang, J. W., Cheng, J. S., Zhou, I. Y., Cheung, M. M., and Wu, E. X. (2013). Noninvasive fMRI investigation of interaural level difference processing in the rat auditory subcortex. PLoS One 8:e70706. doi: 10.1371/journal.pone. 0070706

Lau, C., Zhang, J. W., McPherson, B., Pienkowski, M., and Wu, E. X. (2015b). Long-term, passive exposure to non-traumatic acoustic noise induces neural adaptation in the adult rat medial geniculate body and auditory cortex. Neuroimage 107, 1-9. doi: 10.1016/j.neuroimage.2014.11.048

Lau, C., Zhou, I. Y., Cheung, M. M., Chan, K. C., and Wu, E. X. (2011). BOLD temporal dynamics of rat superior colliculus and lateral geniculate nucleus following short duration visual stimulation. PLoS One 6:e18914. doi: 10.1371/ journal.pone.0018914

Lein, E. S., Hawrylycz, M. J., Ao, N., Ayres, M., Bensinger, A., Bernard, A., et al. (2007). Genome-wide atlas of gene expression in the adult mouse brain. Nature $445,168-176$. doi: $10.1038 /$ nature 05453

Li, L., and Kelly, J. B. (1992). Inhibitory influence of the dorsal nucleus of the lateral lemniscus on binaural responses in the rat's inferior colliculus. J. Neurosci. 12, 4530-4539. doi: 10.1523/jneurosci.12-11-04530.1992

Liberman, M. C., Liberman, L. D., and Maison, S. F. (2015). Chronic conductive hearing loss leads to cochlear degeneration. PLoS One 10:e0142341. doi: 10. 1371/journal.pone.0142341

Liu, B., Feng, Y., Yang, M., Chen, J. Y., Li, J., Huang, Z. C., et al. (2015). Functional connectivity in patients with sensorineural hearing loss using resting-state MRI. Am. J. Audiol. 24, 145-152. doi: 10.1044/2015_AJA-13-0068

Luan, Y., Wang, C., Jiao, Y., Tang, T., Zhang, J., Lu, C., et al. (2020). Abnormal functional connectivity and degree centrality in anterior cingulate cortex in patients with long-term sensorineural hearing loss. Brain Imaging Behav. 14, 682-695. doi: 10.1007/s11682-018-0004-0

Luan, Y., Wang, C., Jiao, Y., Tang, T., Zhang, J., and Teng, G. J. (2019). Dysconnectivity of multiple resting-state networks associated with higher-order functions in sensorineural hearing loss. Front. Neurosci. 13:55. doi: 10.3389/ fnins.2019.00055

Malmierca, M. S. (2003). The structure and physiology of the rat auditory system: an overview. Int. Rev. Neurobiol. 56, 147-211. doi: 10.1016/s0074-7742(03) 56005-6

Manno, F. A. M., Isla, A. G., Manno, S. H. C., Ahmed, I., Cheng, S. H., Barrios, F. A., et al. (2019). Early stage alterations in white matter and decreased functional interhemispheric hippocampal connectivity in the $3 \times \mathrm{xTg}$ mouse model of Alzheimer's disease. Front. Aging Neurosci. 11:39. doi: 10.3389/fnagi. 2019.00039

Manno, F. A. M., Pan, L., Mao, Y., Su, Y., Manno, S. H. C., Cheng, S. H., et al. (2020). Assessing the autonomic and behavioral effects of passive motion in rats using elevator vertical motion and ferris-wheel rotation. J. Vis. Exp. doi: $10.3791 / 59837$

Manno, F. A. M., Rodríguez-Cruces, R., Kumar, R., Ratnanather, J. T., and Lau, C. (2021). Hearing loss impacts gray and white matter across the lifespan: systematic review, meta-analysis and meta-regression. Neuroimage 231:117826. doi: 10.1016/j.neuroimage.2021.117826

McCutcheon, J. E., and Marinelli, M. (2009). Age matters. Eur. J. Neurosci. 29, 997-1014. doi: 10.1111/j.1460-9568.2009.06648.x

Osen, K. K., Imad, J., Wennberg, A. E., Papp, E. A., and Leergaard, T. B. (2019). Waxholm space atlas of the rat brain auditory system: three-dimensional delineations based on structural and diffusion tensor magnetic resonance imaging. Neuroimage 199, 38-56. doi: 10.1016/j.neuroimage.2019.05.016

Pajevic, S., and Pierpaoli, C. (1999). Color schemes to represent the orientation of anisotropic tissues from diffusion tensor data: application to white matter fiber tract mapping in the human brain. Magn. Reson. Med. 42, 526-540. doi: 10.1002/(sici)1522-2594(199909)42:3<526::aid-mrm15>3.0.co;2-j

Paxinos, G., and Watson, C. (2004). The Rat Brain in Stereotaxic Coordinates, 5th Edn. Cambridge, MA: Elsevier.

Propst, E. J., Greinwald, J. H., and Schmithorst, V. (2010). Neuroanatomic differences in children with unilateral sensorineural hearing loss detected using 
functional magnetic resonance imaging. Arch. Otolaryngol. Head Neck Surg. 136, 22-26. doi: 10.1001/archoto.2009.208

Puschmann, S., and Thiel, C. M. (2017). Changed crossmodal functional connectivity in older adults with hearing loss. Cortex 86, 109-122. doi: 10.1016/ j.cortex.2016.10.014

Rosen, M. J., Sarro, E. C., Kelly, J. B., and Sanes, D. H. (2012). Diminished behavioral and neural sensitivity to sound modulation is associated with moderate developmental hearing loss. PLoS One 7:e41514. doi: 10.1371/journal. pone. 0041514

Rybalko, N., Mitrovic, D., Šuta, D., Bureš, Z., Popelář, J., and Syka, J. (2019). Behavioral evaluation of auditory function abnormalities in adult rats with normal hearing thresholds that were exposed to noise during early development. Physiol. Behav. 210:112620. doi: 10.1016/j.physbeh.2019.112620

Salvi, R., Auerbach, B. D., Lau, C., Chen, Y. C., Manohar, S., Liu, X., et al. (2020). "Functional neuroanatomy of salicylate- and noise-induced tinnitus and hyperacusis", in Current Topics in Behavioral Neurosciences (Berlin, Heidelberg: Springer). doi: 10.1007/7854_2020_156 Available online at: https: //link.springer.com/chapter/10.1007\%2F7854_2020_156\#citeas

Scheffler, K., Bilecen, D., Schmid, N., Tschopp, K., and Seelig, J. (1998). Auditory cortical responses in hearing subjects and unilateral deaf patients as detected by functional magnetic resonance imaging. Cereb. Cortex 8, 156-163. doi: 10.1093/ cercor/8.2.156

Schmidt, S. A., Akrofi, K., Carpenter-Thompson, J. R., and Husain, F. T. (2013). Default mode, dorsal attention and auditory resting state networks exhibit differential functional connectivity in tinnitus and hearing loss. PLoS One 8:e76488. doi: 10.1371/journal.pone.0076488

Sohmer, H., Freeman, S., Friedman, I., and Lidan, D. (1991). Auditory brainstem response (ABR) latency shifts in animal models of various types of conductive and sensori-neural hearing losses. Acta Otolaryngol. 111, 206-211. doi: 10.3109/ 00016489109137376

Sohmer, H., and Friedman, I. (1992). Prolonged conductive hearing loss in rat pups causes shorter brainstem transmission time. Hear Res. 61, 189-196. doi: 10.1016/0378-5955(92)90050-w

Sun, W., Deng, A., Jayaram, A., and Gibson, B. (2012). Noise exposure enhances auditory cortex responses related to hyperacusis behavior. Brain Res. 1485, 108-116. doi: 10.1016/j.brainres.2012.02.008

Takesian, A. E., Kotak, V. C., and Sanes, D. H. (2012). Age-dependent effect of hearing loss on cortical inhibitory synapse function. J. Neurophysiol. 107, 937-947. doi: 10.1152/jn.00515.2011

Tibbetts, K., Ead, B., Umansky, A., Coalson, R., Schlaggar, B. L., Firszt, J. B., et al. (2011). Interregional brain interactions in children with unilateral hearing loss. Otolaryngol. Head Neck Surg. 144, 602-611. doi: 10.1177/0194599810394954

Tucci, D. L., Cant, N. B., and Durham, D. (1999). Conductive hearing loss results in a decrease in central auditory system activity in the young gerbil. Laryngoscope 109, 1359-1371. doi: 10.1097/00005537-199909000-00001

von Trapp, G., Aloni, I., Young, S., Semple, M. N., and Sanes, D. H. (2017). Developmental hearing loss impedes auditory task learning and performance in gerbils. Hear. Res. 347, 3-10. doi: 10.1016/j.heares.2016.07.020

Wang, S., Chen, B., Yu, Y., Yang, H., Cui, W., Li, J., et al. (2019). Alterations of structural and functional connectivity in profound sensorineural hearing loss infants within an early sensitive period: a combined DTI and fMRI study. Dev. Cogn. Neurosci. 38:100654. doi: 10.1016/j.dcn.2019.10 0654

Willcox, T. O., and Artz, G. J. (2007). "Auditory system disorders (Chapter 26)," in Neurology and Clinical Neuroscience, ed. A. H. V. Schapira (Amsterdam: Elsevier), 329-335.

Winer, J. A., and Schreiner, C. E. (2005). "The central auditory system: a functional analysis," in The Inferior Colliculus, eds J. A. Winer and C. E. Schreiner (New York, NY: Springer), doi: 10.1007/0-387-27083-3_1
Wolak, T., Cieśla, K., Pluta, A., Włodarczyk, E., Biswal, B., and Skarżyński, H. (2019). Altered functional connectivity in patients with sloping sensorineural hearing loss. Front. Hum. Neurosci. 13:284. doi: 10.3389/fnhum.2019.00284

Wong, E., Radziwon, K., Chen, G. D., Liu, X., Manno, F. A. M., Manno, S. H. C., et al. (2020). Functional magnetic resonance imaging of enhanced central auditory gain and electrophysiological correlates in a behavioral model of hyperacusis. Hear. Res. 389:107908. doi: 10.1016/j.heares.2020.107908

Wong, E., Yang, B., Du, L., Ho, W. H., Lau, C., Ke, Y., et al. (2017). The multi-level impact of chronic intermittent hypoxia on central auditory processing. Neuroimage 156, 232-239. doi: 10.1016/j.neuroimage.2017.0 5.036

Wu, M. F., Krueger, J., Ison, J. R., and Gerrard, R. L. (1984). Startle reflex inhibition in the rat: its persistence after extended repetition of the inhibitory stimulus. J. Exp. Psychol. Anim. Behav. Process. 10, 221-228. doi: 10.1037/0097-7403.10. 2.221

Xia, S., Song, T., Che, J., Li, Q., Chai, C., Zheng, M., et al. (2017). Altered brain functional activity in infants with congenital bilateral severe sensorineural hearing loss: a resting-state functional MRI study under sedation. Neural Plast. 2017:8986362.

Xu, X. M., Jiao, Y., Tang, T. Y., Lu, C. Q., Zhang, J., Salvi, R., et al. (2019). Altered spatial and temporal brain connectivity in the salience network of sensorineural hearing loss and tinnitus. Front. Neurosci. 13:246. doi: 10.3389/ fnins.2019.00246

Yang, B., Wong, E., Ho, W. H., Lau, C., Chan, Y. S., and Wu, E. X. (2018). Reduction of sound-evoked midbrain responses observed by functional magnetic resonance imaging following acute acoustic noise exposure. J. Acoust. Soc. Am. 143:2184. doi: 10.1121/1.5030920

Yao, J. D., and Sanes, D. H. (2018). Developmental deprivation-induced perceptual and cortical processing deficits in awake-behaving animals. Elife 6:7. doi: 10. 7554/eLife.33891

Ye, Y., Ihlefeld, A., and Rosen, M. J. (2021). Conductive hearing loss during development does not appreciably alter the sharpness of cochlear tuning. Sci. Rep. 11:3955. doi: 10.1038/s41598-021-83115-1

Zhang, D. X., Li, L., Kelly, J. B., and Wu, S. H. (1998). GABAergic projections from the lateral lemniscus to the inferior colliculus of the rat. Hear. Res. 117, 1-12. doi: 10.1016/s0378-5955(97)00202-5

Zhang, J., van Zijl, P. C., and Mori, S. (2006). Image contrast using the secondary and tertiary eigenvectors in diffusion tensor imaging. Magn. Reson. Med. 55, 439-449. doi: 10.1002/mrm.20767

Zhou, I. Y., Liang, Y. X., Chan, R. W., Gao, P. P., Cheng, J. S., Hu, Y., et al. (2014). Brain resting-state functional MRI connectivity: morphological foundation and plasticity. Neuroimage 84, 1-10. doi: 10.1016/j.neuroimage.2013.08.037

Conflict of Interest: The authors declare that the research was conducted in the absence of any commercial or financial relationships that could be construed as a potential conflict of interest.

Publisher's Note: All claims expressed in this article are solely those of the authors and do not necessarily represent those of their affiliated organizations, or those of the publisher, the editors and the reviewers. Any product that may be evaluated in this article, or claim that may be made by its manufacturer, is not guaranteed or endorsed by the publisher.

Copyright $\odot 2021$ Manno, An, Kumar, Wu, He, Feng and Lau. This is an open-access article distributed under the terms of the Creative Commons Attribution License (CC BY). The use, distribution or reproduction in other forums is permitted, provided the original author(s) and the copyright owner(s) are credited and that the original publication in this journal is cited, in accordance with accepted academic practice. No use, distribution or reproduction is permitted which does not comply with these terms. 